\title{
Erratum: Optimal and secure measurement protocols for quantum sensor networks [Phys. Rev. A 97, 042337 (2018)]
}

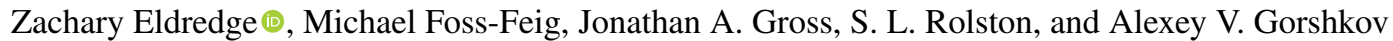

(Received 4 January 2022; published 24 January 2022)

DOI: 10.1103/PhysRevA.105.019903

Section IV B 1 contains an error in the probability distributions defined in Eq. (50). Specifically, the probability distribution is only well defined in the case where $\alpha_{j} \in\{1,0,-1\}$ for all $j$ s; otherwise, at least, one "probability" is negative. Proper probability distributions and, therefore, valid protocols may be derived using the methods developed in Refs. [1,2]. In particular, the set of states considered in Eqs. (46) and (47) of our paper are the same states used in both these works. Therefore, the protocols derived in Sec. V of Ref. [1] and in Ref. [2] are valid in our setting as well.

This error does not affect any of the main conclusions of the article.

We thank A. Ehrenberg and J. Bringewatt for bringing it to our attention.

[1] J. A. Gross and C. M. Caves, J. Phys. A: Math. Theor. 54, 014001 (2021).

[2] A. Ehrenberg, J. Bringewatt, and A. V. Gorshkov, arXiv:2110.07613. 